\title{
RELAXATION OF FREE-DISCONTINUITY ENERGIES WITH OBSTACLES
}

\author{
Matteo Focardi ${ }^{1}$ and Maria Stella Gelli ${ }^{2}$
}

\begin{abstract}
Given a Borel function $\psi$ defined on a bounded open set $\Omega$ with Lipschitz boundary and $\varphi \in L^{1}\left(\partial \Omega, \mathcal{H}^{n-1}\right)$, we prove an explicit representation formula for the $L^{1}$ lower semicontinuous envelope of Mumford-Shah type functionals with the obstacle constraint $u^{+} \geq \psi \mathcal{H}^{n-1}$ a.e. on $\Omega$ and the Dirichlet boundary condition $u=\varphi$ on $\partial \Omega$.
\end{abstract}

Mathematics Subject Classification. 49J45, 74R10.

Received December 12, 2006. Revised May 22, 2007.

Published online February 7, 2008.

\section{INTRODUCTION}

Weak formulations of fracture mechanics theories for brittle hyperelastic media have been studied in the last years in the framework of free-discontinuity problems (see [1,10] and [2] for a more exhaustive list of references). In these models the state of a brittle body is described by a pair displacement-crack with total energy given by the sum of a bulk and a surface term, related to the (approximate) gradient and the set of (approximate) discontinuities of the deformation, respectively.

In particular, homogenization of brittle media with reinforcements may involve minimum problems for freediscontinuity energies with an obstacle condition. In case of bodies with a periodic distribution of perforations, intended in the sense of holes on which a Dirichlet or a unilateral obstacle condition is imposed, one is interested in analyzing the behaviour of the energy as the diameter of the perforations tends to 0 .

In case of antiplane setting and selecting the Mumford-Shah energy as a prototype, one investigates the asymptotics as $\varepsilon$ tends to 0 of Dirichlet boundary value problems for functionals of the type

$$
\int_{\Omega}|\nabla u(x)|^{p} \mathrm{~d} x+\mathcal{H}^{n-1}\left(S_{u}\right)+\text { lower order terms } \quad u^{+} \geq 0 \mathcal{H}^{n-1} \text { a.e. on } \mathbf{E}_{\varepsilon}
$$

where the open set $\Omega \subseteq \mathbf{R}^{n}$ represents a section in the cylindrical reference configuration of the body $\Omega \times \mathbf{R}$, $u \in G S B V(\Omega)$ is the antiplane displacement, and the set $\mathbf{E}_{\varepsilon}$ is obtained periodically perforating the domain $\Omega$ with a rescaled copy of the reference hole $E$.

We remark that the formulation of the obstacle condition in (1.1) as an $\mathcal{H}^{n-1}$ constraint is consistent with perforations $\mathcal{L}^{n}$ negligible, and it is a natural generalization for such sets of the usual unilateral inequality in the $\mathcal{L}^{n}$ sense (see Rem. $4.2[15]$ ).

\footnotetext{
Keywords and phrases. Obstacle problems, Mumford-Shah energy, relaxation.

1 Dip. Mat. "U. Dini", V.le Morgagni, 67/a, 50134 Firenze, Italy; focardi@math.unifi.it

2 Dip. Mat. "L. Tonelli", L.go Bruno Pontecorvo, 5, 56127 Pisa, Italy; gelli@dm.unipi.it
} 
The homogenization problem above was addressed in the paper [15] via $\Gamma$-convergence methods. The convergence of the minimum problems associated to (1.1) to the corresponding problem for the $\Gamma$-limit is a byproduct of such analysis. The coercivity of functionals as in (1.1) is ensured by a well known result of Ambrosio (see Th. 2.1), instead the $L^{1}$ lower semicontinuity of free-discontinuity energies subject to an $\mathcal{H}^{n-1}$ constraint has to be investigated.

In this paper we characterize the relaxed functional associated to an energy as in (1.1) under a general unilateral constraint. Namely, given a Borel function $\psi: \Omega \rightarrow \mathbf{R} \cup\{ \pm \infty\}, p>1$, we consider the functional

$$
F_{\psi}(u, \Omega)=\int_{\Omega}|\nabla u|^{p} \mathrm{~d} x+\mathcal{H}^{n-1}\left(S_{u}\right) \quad \text { if } u \in G S B V(\Omega), u^{+} \geq \psi \mathcal{H}^{n-1} \text { a.e. on } \Omega
$$

and $+\infty$ otherwise in $L^{1}(\Omega)$.

In order to deal with this problem we introduce a variational measure $\sigma$ following the approach of De Giorgi for parametric Plateau problems with an obstacle (see Defs. (3.1), (3.2)). The main result proved in this paper is that the relaxed functional of $F_{\psi}$ can be written in the form

$$
\mathcal{F}_{\psi}(u, \Omega)=\int_{\Omega}|\nabla u|^{p} \mathrm{~d} x+\mathcal{H}^{n-1}\left(S_{u}\right)+\frac{1}{2} \sigma\left(\left\{x \in S_{u}: u^{+}(x)<\psi(x)\right\}\right)+\sigma\left(\left\{x \in \Omega \backslash S_{u}: u^{+}(x)<\psi(x)\right\}\right)
$$

if $u \in G S B V(\Omega),+\infty$ otherwise in $L^{1}(\Omega)$.

In particular, we show that the measure $\sigma$ introduced above coincides with the analogous measure originally defined by De Giorgi for minimal surfaces with obstacles (see Sects. 2.3 and 3 for more exhaustive details).

An outline of the paper is as follows. In Section 2 we review some prerequisites needed in the sequel: We recall some properties of sets with finite perimeter, $B V$ functions and De Giorgi's measure $\sigma$. In Section 3 we introduce and study the properties of a variational measure which is naturally involved in the relaxation process. In particular, we compare it with De Giorgi's one. In Section 4 we state and prove the main result justifying the relaxation formula above. The result is shown to be consistent with the addition of a Dirichlet boundary condition in Section 5. All results illustrated for the Mumford-Shah energy are extended in Section 6 to more general free-discontinuity energies.

\section{NotATION AND PRELIMINARIES}

In the sequel $n \geq 1$ will be a fixed integer, and $p \in(1,+\infty)$ will be a fixed exponent.

\subsection{Relaxation}

We recall the notion of relaxation of a functional $F: X \rightarrow[0,+\infty]$ in a generic metric space $(X, d)$ endowed with the topology induced by $d$ (see $[5,9]$ ). The relaxed functional $\bar{F}: X \rightarrow[0,+\infty]$ is the lower semicontinuous envelope of $F$, that is

$$
\bar{F}(u)=\sup \{G(u): G \leq F, G d \text {-lower semicontinuous }\}
$$

A different characterization holds for $\bar{F}$, namely

$$
\bar{F}(u)=\inf \left\{\liminf _{j \rightarrow+\infty} F\left(u_{j}\right): u_{j} \rightarrow u\right\} .
$$

Thus, given a candidate $\mathcal{F}$ for the lower semicontinuous envelope of $F$, to show that it equals $\bar{F}$ it suffices to prove the following two inequalities:

(i) (lower bound) for every $\left(u_{j}\right)$ converging to $u$ in $X$, we have $\liminf _{j} F\left(u_{j}\right) \geq \mathcal{F}(u)$;

(ii) (upper bound) there exists $\left(u_{j}\right)$ converging to $u$ in $X$ such that $\lim \sup _{j} F\left(u_{j}\right) \leq \mathcal{F}(u)$. 


\subsection{BV functions}

In this subsection we recall some basic definitions and results on sets of finite perimeter, BV, SBV and GSBV functions. We refer to the book [2] for all the results used throughout the whole paper, for which we will give a precise reference.

Let $A \subseteq \mathbf{R}^{n}$ be an open set, for every $u \in L^{1}(A)$ and $x \in A$, we define

$$
\begin{aligned}
& u^{+}(x)=\inf \left\{t \in \mathbf{R}: \lim _{r \rightarrow 0^{+}} r^{-n} \mathcal{L}^{n}\left(\left\{y \in B_{r}(x): u(y)>t\right)\right\}=0\right\} \\
& u^{-}(x)=\sup \left\{t \in \mathbf{R}: \lim _{r \rightarrow 0^{+}} r^{-n} \mathcal{L}^{n}\left(\left\{y \in B_{r}(x): u(y)<t\right)\right\}=0\right\},
\end{aligned}
$$

with the convention $\inf \emptyset=+\infty$ and $\sup \emptyset=-\infty$. We remark that $u^{+}, u^{-}$are Borel functions uniquely determined by the $\mathcal{L}^{n}$-equivalence class of $u$. If $u^{+}(x)=u^{-}(x)$ the common value is denoted by $\tilde{u}(x)$ or ap- $\lim _{y \rightarrow x} u(y)$ and it is said to be the approximate limit of $u$ in $x$.

In particular, for every $\mathcal{L}^{n}$ measurable set $E \subseteq \mathbf{R}^{n}$ it holds $\left(\chi_{E}\right)^{+}=\chi_{E^{+}}$, where

$$
E^{+}=\left\{x \in \mathbf{R}^{n}: \limsup _{r \rightarrow 0^{+}} r^{-n} \mathcal{L}^{n}\left(E \cap B_{r}(x)\right)>0\right\} .
$$

We remark that for any $u \in L^{1}(A)$ and $s \leq t$, it holds

$$
\{x \in A: u(x) \geq s\}^{+} \supseteq\left\{x \in A: u^{+}(x) \geq t\right\} .
$$

The set $S_{u}=\left\{x \in A: u^{-}(x)<u^{+}(x)\right\}$ is called the set of approximate discontinuity points of $u$ and it is well known that $\mathcal{L}^{n}\left(S_{u}\right)=0$. Let $x \in A \backslash S_{u}$ be such that $\tilde{u}(x) \in \mathbf{R}$, we say that $u$ is approximately differentiable at $x$ if there exists $L \in \mathbf{R}^{n}$ such that

$$
\operatorname{ap}-\lim _{y \rightarrow x} \frac{|u(y)-\tilde{u}(x)-L(y-x)|}{|y-x|}=0
$$

If $u$ is approximately differentiable at a point $x$, the vector $L$ uniquely determined by (2.2), will be denoted by $\nabla u(x)$ and will be called the approximate gradient of $u$ at $x$.

A function $u \in L^{1}(A)$ is said to be of Bounded Variation in $A$, in short $u \in B V(A)$, if its distributional derivative $D u$ is a $\mathbf{R}^{n}$-valued finite Radon measure on $A$ with mass $\|D u\|(A)$, called the total variation of $u$ on $A$. If $u \in B V(A)$ denote by $D^{a} u, D^{s} u$ the absolutely and singular part of the Lebesgue's decomposition of $D u$ w.r.t. $\mathcal{L}^{n}\llcorner A$, respectively. Then $u$ turns out to be approximately differentiable a.e. on $A$ (see Ths. 3.83 $[2]), S_{u}$ to be countably $\mathcal{H}^{n-1}$-rectifiable (see Th. $3.78[2]$ ), and the values $u^{+}(x), u^{-}(x)$ are finite and specified $\mathcal{H}^{n-1}$ a.e. in $A$ (see Rem. $3.79[2]$ ). Moreover, it holds

$$
D^{a} u=\nabla u \mathcal{L}^{n}\left\llcorner A, \quad D^{s} u\left\llcorner S_{u}=\left(u^{+}-u^{-}\right) \nu_{u} \mathcal{H}^{n-1}\left\llcorner S_{u},\right.\right.\right.
$$

where $\nu_{u} \in \mathbf{R}^{n}$ is an orientation for $S_{u}$.

We say that a $\mathcal{L}^{n}$ measurable set $E \subseteq \mathbf{R}^{n}$ is of finite perimeter in $A$ if $\chi_{E} \in B V(A)$, and we call the total variation of $\chi_{E}$ in $A$ the perimeter of $E$ in $A$, denoting it by $\operatorname{Per}(E, A)$ and simply by $\operatorname{Per}(E)$ if $A \equiv \mathbf{R}^{n}$. Setting for $t \in[0,1]$

$$
E^{t}=\left\{x \in \mathbf{R}^{n}: \lim _{r \rightarrow 0^{+}} \frac{\mathcal{L}^{n}\left(E \cap B_{r}(x)\right)}{\omega_{n} r^{n}}=t\right\},
$$

and $\partial^{*} E=E \backslash\left(E^{1} \cup E^{0}\right)$, it is well known that the set $\partial^{*} E$ is countably $\mathcal{H}^{n-1}$-rectifiable, and letting $\nu_{\partial^{*} E}$ be an orientation for it we have $D \chi_{E}=D \chi_{E}\left\llcorner\partial^{*} E=\nu_{\partial{ }^{*}} \mathcal{H}^{n-1}\left\llcorner\partial^{*} E\right.\right.$ (see Th. 3.59 [2]).

We say that $u \in B V(A)$ is a Special Function of Bounded Variation in $A$ if $D^{s} u \equiv D^{j} u$ on $A$, in short $u \in S B V(A)$. 
We say that $u \in L^{1}(A)$ is a Generalized Special Function of Bounded Variation in A, in short $u \in G S B V(A)$, if for every $M>0$ the truncated function $(u \wedge M) \vee(-M) \in S B V(A)$.

Functions in $G S B V$ inherit from $B V$ ones many properties: a generalized distributional derivative can be defined, they are approximately differentiable a.e. on $A$, and $S_{u}$ turns out to be countably $\mathcal{H}^{n-1}$-rectifiable (see Th. $4.34[2])$.

The space $(G) S B V$ has been introduced by De Giorgi and Ambrosio [13] in connection with the weak formulation of the image segmentation model proposed by Mumford and Shah (see [18]). If $u \in G S B V(A)$ and $p \in(1,+\infty)$ the Mumford-Shah energy of $u$ is defined as

$$
M S_{p}(u, A)=\int_{A}|\nabla u|^{p} \mathrm{~d} x+\mathcal{H}^{n-1}\left(S_{u}\right) .
$$

We recall the $G S B V$ compactness theorem due to Ambrosio in a form needed for our purposes (see Ths. 4.8 and $5.22[2])$.

Theorem 2.1. Let $\left(u_{j}\right) \subset G S B V(A)$ and assume that for some $p \in(1,+\infty)$

$$
\sup _{j}\left(M S_{p}\left(u_{j}, A\right)+\left\|u_{j}\right\|_{L^{1}(A)}\right)<+\infty .
$$

Then, there exist a subsequence $\left(u_{j_{k}}\right)$ and a function $u \in G S B V(A)$ such that $u_{j_{k}} \rightarrow u$ a.e. in $A, \nabla u_{j_{k}} \rightarrow \nabla u$ weakly in $L^{p}\left(A ; \mathbf{R}^{n}\right), D^{s} u_{j_{k}}\left\llcorner S_{u_{j_{k}}} \rightarrow D^{s} u\left\llcorner S_{u}\right.\right.$ weakly-* in the sense of measures.

In particular, if $\sup _{j}\left\|u_{j}\right\|_{L^{\infty}(A)}<+\infty$ then the cluster point u belongs to $S B V(A)$.

Eventually, if $\varphi: \mathbf{R}^{n} \rightarrow[0,+\infty)$ is a norm, then

$$
\int_{S_{u}} \varphi\left(\nu_{u}\right) \mathrm{d} \mathcal{H}^{n-1} \leq \liminf _{k} \int_{S_{u_{j_{k}}}} \varphi\left(\nu_{u_{j_{k}}}\right) \mathrm{d} \mathcal{H}^{n-1}
$$

Eventually, in case $u \in G S B V(A)$ and $M S_{p}(u, A)<+\infty$ the values $u^{+}(x), u^{-}(x)$ are finite and specified $\mathcal{H}^{n-1}$ a.e. in $A$ (see Th. $4.40[2]$ ).

To conclude the preliminaries on $G S B V$ functions we recall their characterization via restrictions to onedimensional subspaces. For more details on the so called "slicing techniques" we refer both to Section 3.11 [2] and Chapter 4 [4].

Let $\xi \in \mathbf{S}^{n-1}$ be a fixed direction, denote by $\Pi^{\xi}$ the orthogonal space to $\xi$. If $y \in \Pi^{\xi}$ and $E \subset \mathbf{R}^{n}$ define $E_{y}^{\xi}=\{t \in \mathbf{R}: y+t \xi \in E\}$ and $E_{\xi}=\left\{y \in \Pi^{\xi}: E_{y}^{\xi} \neq \emptyset\right\}$. Moreover, given $g: E \rightarrow \mathbf{R}$ define, for any $y \in E_{\xi}$, $g_{\xi, y}: E_{y}^{\xi} \rightarrow \mathbf{R}$ by $g_{\xi, y}(t):=g(y+t \xi)$.

Theorem 2.2. Let $u \in G S B V(A)$, then $u_{\xi, y} \in G S B V\left(A_{y}^{\xi}\right)$ for all $\xi \in \mathbf{S}^{n-1}$ and $\mathcal{H}^{n-1}$ a.e. $y \in A_{\xi}$. For such y we have:

(i) $u_{\xi, y}(t)=\nabla u(y+t \xi) \xi$ for $\mathcal{L}^{1}$ a.e. $t \in A_{y}^{\xi}$;

(ii) $S_{u_{\xi, y}}=\left(S_{u}\right)_{y}^{\xi}$;

(iii) $u_{\xi, y}^{ \pm}(t)=u^{ \pm}(y+t \xi)$ or $u_{\xi, y}^{ \pm}(t)=u^{\mp}(y+t \xi)$ according to the cases $\left\langle\nu_{u}, \xi\right\rangle>0,\left\langle\nu_{u}, \xi\right\rangle<0$ (the case $\left\langle\nu_{u}, \xi\right\rangle=0$ being negligible).

We conclude the subsection recalling a consequence of the Coarea formula (see Th. 2.93 [2]).

Proposition 2.3. For any $u \in G S B V(A)$, for every $\xi \in \mathbf{S}^{n-1}$ and every open set $A^{\prime} \subseteq A$ it holds

$$
\int_{A^{\prime} \cap S_{u}}\left|\left\langle\nu_{u}(x), \xi\right\rangle\right| \mathrm{d} \mathcal{H}^{n-1}(x)=\int_{A_{\xi}^{\prime}} \mathcal{H}^{0}\left(\left(S_{u}\right)_{y}^{\xi}\right) \mathrm{d} \mathcal{H}^{n-1}(y) .
$$




\subsection{De Giorgi's measure}

In this subsection we recall the definition of an $(n-1)$-dimensional geometric measure which has been introduced in the study of obstacle problems for area-like functionals (see [6-8,12,14,19]).

Following the original definition by De Giorgi, Colombini and Piccinini [14], for any open set $A \subseteq \mathbf{R}^{n}$ and any set $E \subseteq \mathbf{R}^{n}$, we consider the set functions

$$
\sigma^{\varepsilon}(E, A)=\inf \left\{\operatorname{Per}(D, A)+\frac{1}{\varepsilon} \mathcal{L}^{n}(D \cap A): D=D^{+}, \quad D \supseteq E \cap A\right\},
$$

and

$$
\sigma(E, A)=\sup _{\varepsilon>0} \sigma^{\varepsilon}(E, A) .
$$

We collect below some properties of $\sigma$ summarizing Theorems 2.3, 2.7, 2.8 and 4.10 of Chapter 4 [14].

Theorem 2.4. Let $A \subseteq \mathbf{R}^{n}$ be an open set and $E \subseteq \mathbf{R}^{n}$.

(a) $\sigma$ is a regular Borel measure such that

$$
c_{1}(n) \mathcal{H}^{n-1}(E \cap A) \leq \sigma(E, A) \leq c_{2}(n) \mathcal{H}^{n-1}(E \cap A)
$$

for two positive constants $c_{1}, c_{2}$ depending only on $n$.

(b) If $E \subseteq A$, then

$$
\sigma(E, A)=\sigma(E) .
$$

In particular, for every set $F \subseteq \mathbf{R}^{n}$ it holds $\sigma(F, A)=\sigma(F \cap A)$.

(c) If $E$ is a $\mathcal{H}^{n-1}$-rectifiable set, then

$$
\sigma(E, A)=2 \mathcal{H}^{n-1}(E \cap A) .
$$

Remark 2.5. The papers [16,17] study in details the relationship between $\sigma$ and $\mathcal{H}^{n-1}$. In particular, an example disproves the equality in (2.7) in general.

Moreover, the inequality $c_{2}(n) \leq n \omega_{n} / \omega_{n-1}$ is established, with $\omega_{k}$ the $\mathcal{L}^{k}$ measure of the unit ball in $\mathbf{R}^{k}$. A further example shows the optimality of that bound for $n=2$, and some hints are given in order to generalize such a result for arbitrary $n \geq 2$. No lower bound for $c_{1}(n)$ is to our knowledge explicit.

We now state alternative characterizations of $\sigma$, the first proved in [8] the others in [6].

Proposition 2.6. For any open set $A \subseteq \mathbf{R}^{n}$ and any set $E \subseteq \mathbf{R}^{n}$, we have

$$
\begin{aligned}
\sigma(E, A) & =\sup _{\varepsilon>0}\left(\inf \left\{\operatorname{Per}(D, A)+\frac{1}{\varepsilon} \mathcal{L}^{n}(D \cap A): D \text { open, } D \supseteq E \cap A\right\}\right) \\
& =\sup _{\varepsilon>0}\left(\inf \left\{\operatorname{Per}(D, A)+\frac{1}{\varepsilon} \mathcal{L}^{n}(D \cap A): D \mathcal{L}^{n} \text { measurable, } \mathcal{H}^{n-1}\left(E \cap A \backslash D^{+}\right)=0\right\}\right) \\
& =\sup _{\varepsilon>0}\left(\inf \left\{\|D u\|(A)+\frac{1}{\varepsilon} \int_{A}|u| \mathrm{d} x: u \in B V(A), u^{+} \geq 1 \mathcal{H}^{n-1} \text { a.e. on } E \cap A\right\}\right) .
\end{aligned}
$$

Remark 2.7. The first characterization of $\sigma$ provided in Proposition 2.6 entails that for any set $E$ for which $\sigma(E, A)<+\infty$ we can find a family of open sets $\left(D_{\varepsilon}\right)$ admissible for the minimum problems $\sigma^{\varepsilon}(E, A)$ satisfying $\mathcal{L}^{n}\left(D_{\varepsilon}\right)=o\left(\varepsilon^{2}\right)$ and

$$
\sigma(E, A)=\operatorname{Per}\left(D_{\varepsilon}, A\right)+o(1) .
$$

The following result clarifies how De Giorgi's measure $\sigma$ arises in the relaxation of obstacle problems with linear growth (see Th. 3.4 in Chap. 4 [14] and Th. 6.1 [6], moreover Th. 7.1 [6] addresses the case when a Dirichlet boundary datum is added). To avoid technicalities we state it in the simplest case. 
Theorem 2.8. Given an open set $A \subseteq \mathbf{R}^{n}$ and a Borel function $\psi: \mathbf{R}^{n} \rightarrow \mathbf{R} \cup\{ \pm \infty\}$, consider

$$
G_{\psi}(u, A)=\int_{A}|\nabla u| \mathrm{d} x, \text { if } u \in W^{1,1}(A), \tilde{u} \geq \psi \mathcal{H}^{n-1} \text { a.e. on } A,
$$

and $+\infty$ otherwise in $L^{1}(A)$. Then, the lower semicontinuous envelope of $G_{\psi}$ in the $L^{1}$ topology is given by

$$
\mathcal{G}_{\psi}(u, A)=\|D u\|(A)+\int_{A}\left[\left(\psi-u^{+}\right) \vee 0\right] \mathrm{d} \sigma
$$

if $u \in B V(A),+\infty$ otherwise in $L^{1}(A)$.

\section{A variational measure}

In this section we introduce a regular Borel measure on any open set $A \subseteq \mathbf{R}^{n}$ following one of the characterizations of the measure $\sigma$ provided in Proposition 2.6.

According to the definition given by [6], for any $\varepsilon>0$ and for any set $E \subseteq \mathbf{R}^{n}$ we consider the set functions $\sigma_{M S}^{\varepsilon}(E, A)$ defined by

$$
\inf \left\{M S_{p}(u, A)+\frac{1}{\varepsilon} \int_{A}|u|^{p} \mathrm{~d} x: u \in S B V(A), u^{+} \geq 1 \mathcal{H}^{n-1} \text { a.e. on } E \cap A\right\}
$$

and

$$
\sigma_{M S}(E, A)=\sup _{\varepsilon>0} \sigma_{M S}^{\varepsilon}(E, A)
$$

with the convention of dropping the dependence on $A$ when $A=\mathbf{R}^{n}$.

Remark 3.1. Similarly to Remark 2.7 the very definition of $\sigma_{M S}$ entails that for any set $E$ for which $\sigma_{M S}(E, A)<+\infty$ we can find a family of functions $\left(v_{\varepsilon}\right) \subseteq S B V(A)$ admissible for the minimum problems $\sigma_{M S}^{\varepsilon}(E, A)$ satisfying $\left\|v_{\varepsilon}\right\|_{L^{p}(A)}^{p}=o\left(\varepsilon^{2}\right)$ and

$$
\sigma_{M S}(E, A)=M S_{p}\left(v_{\varepsilon}, A\right)+o(1) .
$$

It turns out that the set function $\sigma_{M S}$ introduced above coincides with the measure $\sigma$. To explain this fact we notice that the penalization of the $L^{p}$ norm forces minimizing functions for $\sigma_{M S}^{\varepsilon}(E, A)$ to make a transition from 1 to 0 in a thinner and thinner set enclosing $E$. Therefore the superlinearity in the bulk term makes energetically more convenient for minimizing functions to have a discontinuity in a neighbourhood of $E$ rather than having a high gradient energy. Finally, note that the Mumford-Shah and the total variation functionals coincide on sets of finite perimeter.

Proposition 3.2. For any open set $A \subseteq \mathbf{R}^{n}$, for any set $E \subseteq \mathbf{R}^{n}$, we have

$$
\sigma_{M S}(E, A)=\sigma(E, A) .
$$

Proof. Let $A$ be a fixed open set throughout all the proof. Given a set $E$, taking into account that for any measurable set $D \subseteq \mathbf{R}^{n}$ with finite perimeter which is admissible for $\sigma(E, A)$, the function $u=\chi_{D} \in S B V(A)$ is admissible for $\sigma_{M S}(E, A)$ and $M S_{p}(u, A)=\operatorname{Per}(D, A)$, we have $\sigma_{M S}(E, A) \leq \sigma(E, A)$.

In order to get the opposite inequality it suffices to consider a set $E$ such that $\sigma_{M S}(E, A)<+\infty$. Fixed a family of functions $\left(v_{\varepsilon}\right)$ as in Remark 3.1, the strategy to prove the inequality $\sigma_{M S} \geq \sigma$ relies on finding suitable superlevel sets of $v_{\varepsilon}$ such that their perimeters are bounded above by the Mumford-Shah energies of $v_{\varepsilon}$, their $\mathcal{L}^{n}$ measures are negligible with respect to $\varepsilon$, and the set $E \cap A$ is contained $\mathcal{H}^{n-1}$ a.e. in such superlevel sets. 
Let $\eta>0$, then by Remark 3.1 we can find $v_{\varepsilon} \in S B V(A)$ such that $v_{\varepsilon}^{+}(x) \geq 1 \mathcal{H}^{n-1}$ a.e. on $E \cap A$ and

$$
M S_{p}\left(v_{\varepsilon}, A\right)+\frac{1}{\varepsilon^{2}} \int_{A}\left|v_{\varepsilon}\right|^{p} \mathrm{~d} x \leq \sigma_{M S}(E, A)+\eta .
$$

Up to passing to $0 \vee v_{\varepsilon} \wedge 1$ we may also assume that $0 \leq v_{\varepsilon} \leq 1$. By the $B V$ Coarea formula (see Th. 3.40 [2]) we may choose $z_{\varepsilon} \in\left(\varepsilon^{\frac{1}{2 p}}, 1\right)$ such that

$$
\left(1-\varepsilon^{\frac{1}{2 p}}\right) \operatorname{Per}\left(\left\{x \in A: v_{\varepsilon}(x)>z_{\varepsilon}\right\}, A\right) \leq \int_{\varepsilon^{\frac{1}{2 p}}}^{1} \operatorname{Per}\left(\left\{x \in A: v_{\varepsilon}(x)>t\right\}, A\right) \mathrm{d} t \leq\left\|D v_{\varepsilon}\right\|\left(\left\{x \in A: v_{\varepsilon}(x)>\varepsilon^{\frac{1}{2 p}}\right\}\right) .
$$

Letting $D_{\varepsilon}:=\left\{x \in A: v_{\varepsilon}(x)>z_{\varepsilon}\right\}$ and $A_{\varepsilon}:=\left\{x \in A: v_{\varepsilon}(x)>\varepsilon^{\frac{1}{2 p}}\right\}$, Hölder inequality, the fact that $\left|v_{\varepsilon}^{+}(x)-v_{\varepsilon}^{-}(x)\right| \leq 1 \mathcal{H}^{n-1}$ a.e., and (3.4) imply

$$
\left(1-\varepsilon^{\frac{1}{2 p}}\right) \operatorname{Per}\left(D_{\varepsilon}, A\right) \leq \int_{A_{\varepsilon}}\left|\nabla v_{\varepsilon}\right| \mathrm{d} x+\mathcal{H}^{n-1}\left(S_{v_{\varepsilon}}\right) \leq \mathcal{L}^{n}\left(A_{\varepsilon}\right)^{\frac{p-1}{p}}\left\|\nabla v_{\varepsilon}\right\|_{L^{p}(A)}+\mathcal{H}^{n-1}\left(S_{v_{\varepsilon}}\right) .
$$

Moreover, by (3.3)

$$
\mathcal{L}^{n}\left(A_{\varepsilon}\right) \varepsilon^{\frac{1}{2}} \leq \int_{A}\left|v_{\varepsilon}\right|^{p} \mathrm{~d} x \leq\left(\sigma_{M S}(E, A)+\eta\right) \varepsilon^{2},
$$

from which we infer $\mathcal{L}^{n}\left(A_{\varepsilon}\right)=o(\varepsilon)$ and $\mathcal{L}^{n}\left(D_{\varepsilon}\right)=o(\varepsilon)$, and thus $D_{\varepsilon}$ has finite perimeter in $A$.

In particular, using (3.3), for $\varepsilon$ small enough (3.5) rewrites as

$$
\operatorname{Per}\left(D_{\varepsilon}, A\right) \leq \sigma_{M S}(E, A)+2 \eta \text {. }
$$

Furthermore, $D_{\varepsilon}^{+} \supseteq\left\{x \in A: v_{\varepsilon}^{+}(x) \geq 1\right\}$ by (2.1), and thus $\mathcal{H}^{n-1}\left((E \cap A) \backslash D_{\varepsilon}^{+}\right)=0$. Hence, $D_{\varepsilon}$ is admissible for $\sigma^{\varepsilon}(E, A)$ and, taking (3.6) into account, for $\varepsilon$ small enough it holds

$$
\sigma^{\varepsilon}(E, A) \leq \operatorname{Per}\left(D_{\varepsilon}, A\right)+\frac{\mathcal{L}^{n}\left(D_{\varepsilon}\right)}{\varepsilon} \leq \sigma_{M S}(E, A)+3 \eta .
$$

Taking first the supremum on $\varepsilon$ and then letting $\eta \rightarrow 0^{+}$we get the desired inequality.

Remark 3.3. Following [8] and [6] one could equivalently define $\sigma_{M S}(E, A)$ as the supremum of the set functions

$$
\inf \left\{M S_{p}(u, A)+\frac{1}{\varepsilon} \int_{A}|u|^{p} \mathrm{~d} x: u \in S B V(A), u \geq 1 \mathcal{L}^{n} \text { a.e. on an open set } U \supseteq E \cap A\right\} .
$$

Actually, by using the previous proposition and exploiting the equivalence between the two definitions already proven for the measure $\sigma$ one gets that the final measure is the same (see Prop. 2.6).

Remark 3.4. The proof of Proposition 3.2 shows that the measure $\sigma$ coincides also with the one obtained by substituting in definitions (3.1), (3.2) the Mumford-Shah energy with any of the form

$$
\int_{A} f(\nabla u) \mathrm{d} x+\mathcal{H}^{n-1}\left(S_{u} \cap A\right)
$$

where $f: \mathbf{R}^{n} \rightarrow \mathbf{R}$ is such that

$$
c_{1}|\xi|^{p} \leq f(\xi) \leq c_{2}|\xi|^{p}
$$

for every $\xi \in \mathbf{R}^{n}$, for some constants $c_{1}, c_{2}>0$ (see also Sect. 6). 
We now introduce a Borel measure accounting for a generic obstacle. Let $\psi: \mathbf{R}^{n} \rightarrow \mathbf{R} \cup\{ \pm \infty\}, A$ an open set in $\mathbf{R}^{n}$, and $E \subseteq \mathbf{R}^{n}$, for any $\varepsilon>0$ define $\sigma_{M S}^{\varepsilon}(E, A, \psi)$ as

$$
\inf \left\{M S_{p}(u, A)+\frac{1}{\varepsilon} \int_{A}|u|^{p} \mathrm{~d} x: u \in S B V(A), u^{+} \geq \psi \mathcal{H}^{n-1} \text { a.e. on } E \cap A\right\},
$$

and as usual set $\sigma_{M S}(E, A, \psi)=\sup _{\varepsilon>0} \sigma_{M S}^{\varepsilon}(E, A, \psi)$. With a slight abuse of notation when $\psi(x) \equiv c$ we denote $\sigma_{M S}(\cdot, A, \psi)$ simply by $\sigma_{M S}(\cdot, A, c)$. With this notation then $\sigma_{M S}(\cdot, A, 1)=\sigma_{M S}(\cdot, A)$.

Assuming $\psi$ to be a Borel function, one can push forward the arguments used in Proposition 3.2 and prove the following description of $\sigma_{M S}(\cdot, A, \psi)$ on Borel sets.

Proposition 3.5. For any open set $A$, for any Borel function $\psi$, and any Borel set $E \subseteq \mathbf{R}^{n}$ we have

$$
\sigma_{M S}(E, A, \psi)=\sigma_{M S}(\{x \in E: \psi(x)>0\}, A) .
$$

Proof. The open set $A$ and the Borel function $\psi$ will be fixed throughout the whole proof.

Given a Borel set $E$ we first prove that $\sigma_{M S}(E, A, \psi) \geq \sigma_{M S}(\{x \in E: \psi(x)>0\}, A)$. Thus, it is not restrictive to assume $\sigma_{M S}(E, A, \psi)<+\infty$. With $\lambda \in(0,1)$ fixed, we claim that

$$
\sigma_{M S}(E, A, \psi) \geq \sigma_{M S}(\{x \in E: \psi(x)>\lambda\}, A) .
$$

It is clear that the required inequality will easily follow letting $\lambda \rightarrow 0^{+}$and using the fact already proved that $\sigma_{M S}(\cdot, A)$ is a regular Borel measure.

In order to get (3.9) we will exploit the same construction and arguments introduced in the proof of Proposition 3.2 complemented with the Borel regularity assumptions.

Let $\eta>0$ be fixed, reasoning as in Remark 3.1 one can consider functions $w_{\varepsilon} \in S B V(A)$ such that $w_{\varepsilon}^{+}(x) \geq$ $\psi(x) \mathcal{H}^{n-1}$ a.e. on $E \cap A$ and

$$
M S_{p}\left(w_{\varepsilon}, A\right)+\frac{1}{\varepsilon^{2}} \int_{A}\left|w_{\varepsilon}\right|^{p} \mathrm{~d} x \leq \sigma_{M S}(E, A, \psi)+\eta .
$$

Arguing as in the proof of Proposition 3.2 with respect to the functions $v_{\varepsilon}:=0 \vee\left(w_{\varepsilon} / \lambda\right) \wedge 1$, one can find superlevel sets $D_{\varepsilon}=\left\{x \in A: w_{\varepsilon}(x)>z_{\varepsilon}^{\prime}\right\}$ with $z_{\varepsilon}^{\prime} \in\left(\lambda \varepsilon^{\frac{1}{2 p}}, \lambda\right)$ such that

$$
\operatorname{Per}\left(D_{\varepsilon}, A\right)+\frac{1}{\varepsilon} \mathcal{L}^{n}\left(D_{\varepsilon}\right) \leq \sigma_{M S}(E, A, \psi)+3 \eta .
$$

Indeed, taking into account (3.10) and the fact that

$$
\frac{1}{\varepsilon^{2}} \int_{A}\left|v_{\varepsilon}\right|^{p} \mathrm{~d} x \leq \frac{1}{\lambda^{p} \varepsilon^{2}} \int_{A}\left|w_{\varepsilon}\right|^{p} \mathrm{~d} x
$$

if we set $D_{\varepsilon}=\left\{x \in A: v_{\varepsilon}(x)>z_{\varepsilon}\right\}$ with any $z_{\varepsilon} \in\left(\varepsilon^{\frac{1}{2 p}}, 1\right)$, we get $\mathcal{L}^{n}\left(D_{\varepsilon}\right)=o(\varepsilon)$. We now choose $z_{\varepsilon} \in\left(\varepsilon^{\frac{1}{2 p}}, 1\right)$ such that (3.5) holds true for $v_{\varepsilon}$ defined as above. Since $\mathcal{L}^{n}\left(D_{\varepsilon}\right)=o(\varepsilon),\left\|\nabla v_{\varepsilon}\right\|_{L^{p}(A)} \leq \lambda^{-1}\left\|\nabla w_{\varepsilon}\right\|_{L^{p}(A)}$ and $\mathcal{H}^{n-1}\left(S_{v_{\varepsilon}}\right) \leq \mathcal{H}^{n-1}\left(S_{w_{\varepsilon}}\right)$, it is enough to take $z_{\varepsilon}^{\prime}:=\lambda z_{\varepsilon}$ to obtain superlevel sets of the initial functions $w_{\varepsilon}$ with the property (3.11).

Moreover, since for $\mathcal{H}^{n-1}$ a.e. $x \in A \cap\{x \in E: \psi(x)>\lambda\}$ it holds $w_{\varepsilon}^{+}(x) \geq \psi(x)>\lambda$, by definition $v_{\varepsilon}^{+}(x)=1$. Hence, taking (2.1) into account, $D_{\varepsilon}^{+} \supseteq\left\{x \in A: v_{\varepsilon}^{+}(x) \geq 1\right\} \supseteq A \cap\{x \in E: \psi(x)>\lambda\}$ and thus the functions $u_{\varepsilon}=\chi_{D_{\varepsilon}}$ are admissible for $\sigma^{\varepsilon}(\{x \in E: \psi(x)>\lambda\}, A)$. Letting eventually $\eta \rightarrow 0^{+}$in (3.11), we get (3.9).

Notice that the same argument implies that for any positive constant $c$ we have

$$
\sigma_{M S}(E, A, c)=\sigma_{M S}(E, A) .
$$


In order to prove the inverse inequality let us consider a Borel set $E$ such that $\sigma_{M S}(\{x \in E: \psi(x)>0\}, A)<$ $+\infty$. This condition implies at once that the set $A \cap\{x \in E: \psi(x)=+\infty\}$ is $\mathcal{H}^{n-1}$ negligible (see (2.5)). Setting $E_{i}=\{x \in E: i+1 \geq \psi(x)>i\}$ for $i \in \mathbf{N}$, by the standard additivity property of the Borel measure $\sigma_{M S}(\cdot, A)$ we have

$$
\sigma_{M S}(\{x \in E: \psi(x)>0\}, A)=\sum_{i=0}^{+\infty} \sigma_{M S}\left(E_{i}, A\right) .
$$

For $\eta>0$ fixed, let $v_{\varepsilon}^{i}$ be almost optimal for $\sigma_{M S}\left(E_{i}, A, i+1\right)$, that is $\left(v_{\varepsilon}^{i}\right)^{+}(x) \geq i+1 \mathcal{H}^{n-1}$ a.e. on $E_{i}$ and

$$
M S_{p}\left(v_{\varepsilon}^{i}, A\right)+\frac{1}{\varepsilon} \int_{A}\left|v_{\varepsilon}^{i}\right|^{p} \mathrm{~d} x \leq \sigma_{M S}\left(E_{i}, A\right)+\frac{\eta}{2^{i}},
$$

recalling that $\sigma_{M S}\left(E_{i}, A, i+1\right)=\sigma_{M S}\left(E_{i}, A\right)$ by (3.12).

Set $u_{\varepsilon}^{k}:=\sup _{0 \leq i \leq k} v_{\varepsilon}^{i}$. Then $u_{\varepsilon}^{k} \in S B V(A)$ and

$$
M S_{p}\left(u_{\varepsilon}^{k}, A\right)+\frac{1}{\varepsilon} \int_{A}\left|u_{\varepsilon}^{k}\right|^{p} \mathrm{~d} x \leq \sum_{i=0}^{k}\left(M S_{p}\left(v_{\varepsilon}^{i}, A\right)+\frac{1}{\varepsilon} \int_{A}\left|v_{\varepsilon}^{i}\right|^{p} \mathrm{~d} x\right) \leq \sum_{i=0}^{k} \sigma_{M S}\left(E_{i}, A\right)+2 \eta .
$$

In particular, $\left(u_{\varepsilon}^{k}\right)$ is a non-decreasing sequence satisfying the hypotheses of the GSBV compactness Theorem 2.1, so that there exists a function $u_{\varepsilon} \in S B V(A)$ such that $u_{\varepsilon}^{k} \rightarrow u_{\varepsilon}$ in $L^{1}(A)$. Thus, from (3.14) it follows

$$
M S_{p}\left(u_{\varepsilon}, A\right)+\frac{1}{\varepsilon} \int_{A}\left|u_{\varepsilon}\right|^{p} \mathrm{~d} x \leq \liminf _{k \rightarrow+\infty}\left(M S_{p}\left(u_{\varepsilon}^{k}, A\right)+\frac{1}{\varepsilon} \int_{A}\left|u_{\varepsilon}^{k}\right|^{p} \mathrm{~d} x\right) \leq \sigma_{M S}(\{x \in E: \psi(x)>0\}, A)+2 \eta .
$$

Moreover, since $A \cap\{x \in E: \psi(x)>0\}=\cup_{i \geq 0}\left(A \cap E_{i}\right)$, for $\mathcal{H}^{n-1}$ a.e. $z \in A \cap\{x \in E: \psi(x)>0\}$ there exists $i \in \mathbf{N}$ such that $z \in A \cap E_{i}$, and then $u_{\varepsilon}^{+}(z) \geq\left(u_{\varepsilon}^{i+1}\right)^{+}(z) \geq i+1 \geq \psi(z)$. Eventually, $u_{\varepsilon}$ is admissible as a test function for $\sigma_{M S}^{\varepsilon}(E, A, \psi)$ and the inequality follows as usual.

\section{Relaxation Result}

Given an open bounded set $\Omega$ and a Borel function $\psi: \Omega \rightarrow \mathbf{R} \cup\{ \pm \infty\}$, we study the lower semicontinuous envelope of the functional $F_{\psi}: L^{1}(\Omega) \rightarrow[0,+\infty]$ defined by

$$
F_{\psi}(u, \Omega)=\int_{\Omega}|\nabla u|^{p} \mathrm{~d} x+\mathcal{H}^{n-1}\left(S_{u}\right) \quad \text { if } u \in G S B V(\Omega), u^{+} \geq \psi \mathcal{H}^{n-1} \text { a.e. on } \Omega,
$$

and $+\infty$ otherwise in $L^{1}(\Omega)$.

Building on what has been shown in Section 3 we are able to prove the main result of the paper.

Theorem 4.1. Let $F_{\psi}$ be as in (4.1), then its lower semicontinuous envelope in the $L^{1}$ topology is given by

$$
\mathcal{F}_{\psi}(u, \Omega)=\int_{\Omega}|\nabla u|^{p} \mathrm{~d} x+\mathcal{H}^{n-1}\left(S_{u}\right)+\frac{1}{2} \sigma\left(\left\{x \in S_{u}: u^{+}(x)<\psi(x)\right\}\right)+\sigma\left(\left\{x \in \Omega \backslash S_{u}: u^{+}(x)<\psi(x)\right\}\right)
$$

if $u \in G S B V(\Omega),+\infty$ otherwise in $L^{1}(\Omega)$.

In the sequel it is not restrictive to presume the existence of $w \in G S B V(\Omega)$ such that $F_{\psi}(w, \Omega)<+\infty$, being otherwise $F_{\psi} \equiv \mathcal{F}_{\psi} \equiv+\infty$.

For such $w$ 's we have $\{x \in \Omega: \psi(x)=+\infty\} \subseteq\left\{x \in \Omega: w^{+}(x)=+\infty\right\}$, which implies

$$
\mathcal{H}^{n-1}(\{x \in \Omega: \psi(x)=+\infty\})=0
$$

(see Th. $4.40[2])$. 
To prove Theorem 4.1 we address separately the lower and upper bound inequalities.

Proposition 4.2. For every $u$ and $\left(u_{j}\right)$ in $L^{1}(\Omega)$ such that $u_{j} \rightarrow u$ in $L^{1}(\Omega)$ we have

$$
\liminf _{j} F_{\psi}\left(u_{j}, \Omega\right) \geq \mathcal{F}_{\psi}(u, \Omega)
$$

Proof. First notice that we may assume $\liminf { }_{j} F_{\psi}\left(u_{j}, \Omega\right)$ to be finite being the result trivial otherwise; then by Ambrosio's Theorem 2.1 we have $u \in G S B V(\Omega)$. Moreover, we may assume the inferior limit above to be a limit up to extracting a subsequence which we do not relabel for convenience.

We claim the following three estimates to hold true for every open set $A \subseteq \Omega$

$$
\begin{gathered}
\liminf _{j} F_{\psi}\left(u_{j}, A\right) \geq M S_{p}(u, A), \\
\liminf _{j} F_{\psi}\left(u_{j}, A\right) \geq \sigma\left(A \cap\left\{x \in S_{u}: u^{+}(x)<\psi(x)\right\}\right), \\
\liminf _{j} F_{\psi}\left(u_{j}, A\right) \geq \sigma\left(A \cap\left\{x \in \Omega \backslash S_{u}: u^{+}(x)<\psi(x)\right\}\right) .
\end{gathered}
$$

Given them for granted the result follows by standard measure theoretic arguments (see Prop. 1.16 [4]). Indeed, set $\Sigma_{u}=\left\{x \in \Omega: u^{+}(x)<\psi(x)\right\}$, then from (4.4)-(4.6) and taking into account (2.7), for any $\lambda, \mu \in[0,1]$, $\lambda+\mu \leq 1$ it follows

$$
\begin{aligned}
\liminf _{j} F_{\psi}\left(u_{j}, A\right) \geq \lambda \int_{A}|\nabla u|^{p} \mathrm{~d} x+\lambda \mathcal{H}^{n-1}\left(A \cap\left(S_{u} \backslash \Sigma_{u}\right)\right) & \\
& +(\lambda+2 \mu) \mathcal{H}^{n-1}\left(A \cap S_{u} \cap \Sigma_{u}\right)+(1-\lambda-\mu) \sigma\left(A \cap\left(\Sigma_{u} \backslash S_{u}\right)\right) .
\end{aligned}
$$

Being the left hand side above a superadditive set function on disjoint open sets of $\Omega$ and the right hand side sum of orthogonal Radon measures, we can pass to the supremum on $\lambda, \mu$ separately on each term and infer

$$
\begin{gathered}
\liminf _{j} F_{\psi}\left(u_{j}, \Omega\right) \geq \int_{\Omega}|\nabla u|^{p} \mathrm{~d} x+\mathcal{H}^{n-1}\left(S_{u} \backslash \Sigma_{u}\right)+2 \mathcal{H}^{n-1}\left(S_{u} \cap \Sigma_{u}\right)+\sigma\left(\Sigma_{u} \backslash S_{u}\right) \\
=\int_{\Omega}|\nabla u|^{p} \mathrm{~d} x+\mathcal{H}^{n-1}\left(S_{u}\right)+\frac{1}{2} \sigma\left(S_{u} \cap \Sigma_{u}\right)+\sigma\left(\Sigma_{u} \backslash S_{u}\right),
\end{gathered}
$$

which gives the thesis.

Since (4.4) follows immediately by Ambrosio's Theorem 2.1, to conclude the proof we are left with showing the validity of (4.5) and (4.6).

Step 1. Proof of (4.5). We begin with proving the inequality in the one-dimensional case which reads as follows

$$
\liminf _{j} \int_{\Omega}\left|\dot{u}_{j}\right|^{p} \mathrm{~d} t+\mathcal{H}^{0}\left(S_{u_{j}}\right) \geq 2 \mathcal{H}^{0}\left(S_{u} \cap \Sigma_{u}\right) .
$$

We notice that the approximating functions are forced to make a transition from the trace values $u^{ \pm}(\bar{t})$ to the obstacle constraint $\psi(\bar{t})$ in any neighbourhood $I$ of a discontinuity point $\bar{t}$ of $u$ where the constraint is violated, that is $u^{+}(\bar{t})<\psi(\bar{t})$. Hence, to prove the estimate above we will quantify the cost of this transition, and show that it is energetically convenient for the approximating functions to have asymptotically at least 2 discontinuity points in $I$.

Recall that we have assumed

$$
\liminf _{j} \int_{\Omega}\left|\dot{u}_{j}\right|^{p} \mathrm{~d} t+\mathcal{H}^{0}\left(S_{u_{j}}\right)=\lim _{j} \int_{\Omega}\left|\dot{u}_{j}\right|^{p} \mathrm{~d} t+\mathcal{H}^{0}\left(S_{u_{j}}\right) \leq M<+\infty,
$$


which gives $u_{j} \in S B V(\Omega)$ for every $j \in \mathbf{N}$; moreover, for a subsequence not relabeled for convenience, we suppose $u_{j} \rightarrow u \mathcal{L}^{1}$ a.e. in $\Omega$.

We claim that for $j$ sufficiently big

$$
\mathcal{H}^{0}\left(A \cap S_{u_{j}}\right) \geq 2 \mathcal{H}^{0}\left(A \cap S_{u} \cap \Sigma_{u}\right) .
$$

With fixed $\bar{t}$ in the finite set $S_{u} \cap \Sigma_{u}$, there exists $\delta>0$ such that $I_{\delta}=(\bar{t}-\delta, \bar{t}+\delta) \subset \subset A$ and $I_{\delta} \cap S_{u}=\{\bar{t}\}$. Furthermore, being $u$ a Sobolev function on $(\bar{t}-\delta, \bar{t})$ and $(\bar{t}, \bar{t}+\delta)$ separately, we choose $\delta>0$ sufficiently small such that

$$
u(t) \leq u^{+}(\bar{t})+\varepsilon
$$

for every $t \in I_{\delta}$, with $\varepsilon \in\left(0,\left(\psi(\bar{t})-u^{+}(\bar{t})\right) / 4\right)$.

It is clear that (4.8) follows provided we show

$$
\liminf _{j} \mathcal{H}^{0}\left(I_{\delta} \cap S_{u_{j}}\right) \geq 2
$$

Arguing by contradiction we first observe that, up to a subsequence, $I_{\delta} \cap S_{u_{j}}=\left\{t_{j}\right\}$ since by lower semicontinuity $\liminf _{j} \mathcal{H}^{0}\left(I_{\delta} \cap S_{u_{j}}\right) \geq 1$ (see Th. 2.1). In the sequel we show that this implies

$$
\liminf _{j} \int_{I_{\delta}}\left|\dot{u}_{j}\right|^{p} \mathrm{~d} t \geq \delta^{1-p}\left(\frac{\psi(\bar{t})-u^{+}(\bar{t})}{4}\right)^{p}
$$

Select two points $s_{1} \in(\bar{t}-\delta, \bar{t}) \backslash \cup_{j}\left\{t_{j}\right\}$ and $s_{2} \in(\bar{t}, \bar{t}+\delta) \backslash \cup_{j}\left\{t_{j}\right\}$ such that $u_{j}\left(s_{i}\right) \rightarrow u\left(s_{i}\right)$ for $i=1,2$, then for $j$ sufficiently big and $i=1,2$

Then an easy computation yields

$$
u_{j}\left(s_{i}\right) \leq u\left(s_{i}\right)+\varepsilon \leq u^{+}(\bar{t})+2 \varepsilon .
$$

$$
\int_{I_{\delta}}\left|\dot{u}_{j}\right|^{p} \mathrm{dt} \geq \max \left\{\int_{s_{1}}^{\bar{t}}\left|\dot{u}_{j}\right|^{p} \mathrm{~d} t, \int_{\bar{t}}^{s_{2}}\left|\dot{u}_{j}\right|^{p} \mathrm{~d} t\right\} \geq \delta^{1-p}\left(\frac{\psi(\bar{t})-u^{+}(\bar{t})}{4}\right)^{p} .
$$

Indeed, either $t_{j} \neq \bar{t}$ and thus $u_{j}(\bar{t}) \geq \psi(\bar{t})$ or $t_{j}=\bar{t}$ and one between the one sided traces $u_{j}\left(\bar{t}^{ \pm}\right)=\lim _{t \rightarrow \bar{t}^{ \pm}} u_{j}(t)$ equals $u_{j}^{+}(\bar{t})$ which is bigger than or equal to $\psi(\bar{t})$. Assume for instance $t_{j}>\bar{t}$, then $u_{j} \in W^{1, p}\left(s_{1}, \bar{t}\right)$ and by applying Jensen's inequality we infer

$$
\int_{s_{1}}^{\bar{t}}\left|\dot{u}_{j}\right|^{p} \mathrm{~d} t \geq\left|\bar{t}-s_{1}\right|^{1-p}\left(\frac{u_{j}(\bar{t})-u_{j}\left(s_{1}\right)}{2}\right)^{p} \geq \delta^{1-p}\left(\frac{\psi(\bar{t})-u^{+}(\bar{t})}{4}\right)^{p} .
$$

The remaining cases can be worked out similarly (see Fig. 1).

From (4.10) we get a contradiction since (4.7) is violated for $\delta$ sufficiently small. Thus (4.9) holds true and eventually (4.8) follows.

To recover the multi-dimensional setting $n>1$ we use the standard integral geometric reduction technique for which we have introduced some notation in Section 2.2. With fixed $\xi \in \mathbf{S}^{n-1}$, Proposition 2.3 and Fatou's lemma imply for $\mathcal{H}^{n-1}$ a.e. $y \in A_{\xi}$

$$
\liminf _{j} \int_{A_{y}^{\xi}}\left|\left(\dot{u}_{j}\right)_{\xi, y}\right|^{p} \mathrm{~d} t+\mathcal{H}^{0}\left(A_{y}^{\xi} \cap S_{\left(u_{j}\right)_{\xi, y}}\right)<+\infty .
$$

Moreover, Theorem 2.2 and Fubini's theorem imply for $\mathcal{H}^{n-1}$ a.e. $y \in A_{\xi}$

$$
\left(u_{j}\right)_{\xi, y}, u_{\xi, y} \in S B V\left(A_{y}^{\xi}\right),\left(u_{j}\right)_{\xi, y} \rightarrow u_{\xi, y} \text { in } L^{1}\left(A_{y}^{\xi}\right) .
$$



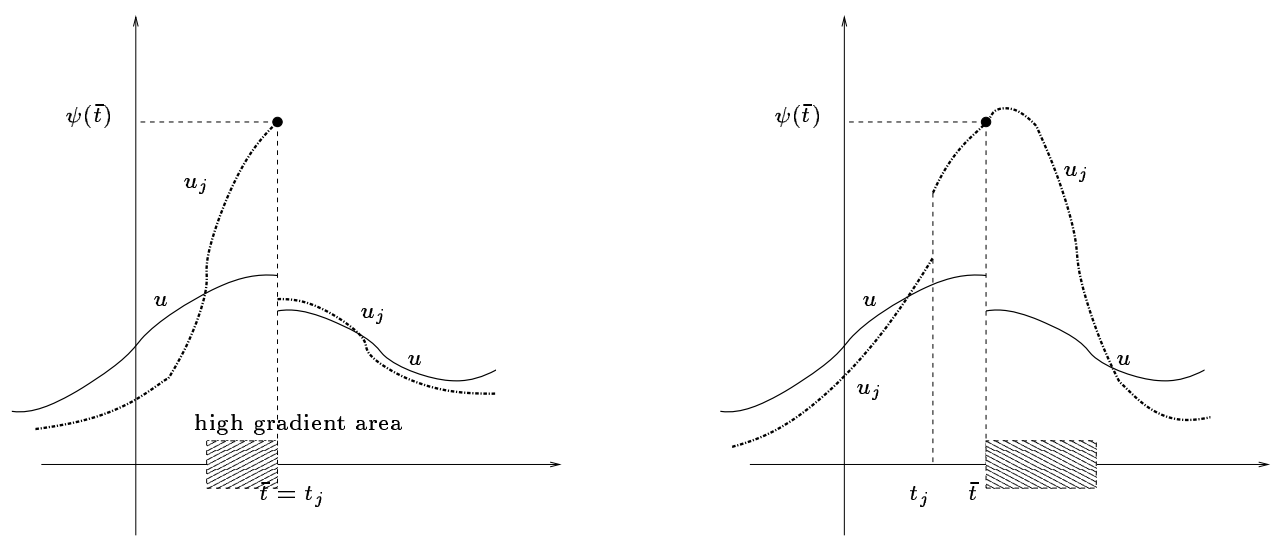

FIGURE 1. Energy concentration of $u_{j}$ according to two possible configurations.

With fixed $y \in A_{\xi}$ satisfying (4.11) and (4.12), we may also suppose $\left(u_{j}\right)_{\xi, y} \rightarrow u_{\xi, y} \mathcal{L}^{1}$ a.e. in $A_{y}^{\xi}$, up to extracting a subsequence. Then, by (4.8) we have

$$
\liminf _{j} \mathcal{H}^{0}\left(A_{y}^{\xi} \cap S_{\left(u_{j}\right)_{\xi, y}}\right) \geq 2 \mathcal{H}^{0}\left(A_{y}^{\xi} \cap S_{u_{\xi, y}} \cap\left(\Sigma_{u}\right)_{y}^{\xi}\right)
$$

which, together with Fatou's lemma and Proposition 2.3, give

$$
\begin{aligned}
& \liminf _{j} F_{\psi}\left(u_{j}, A\right) \geq \int_{A_{\xi}} \liminf _{j}\left(\int_{A_{y}^{\xi}}\left|\left(\dot{u}_{j}\right)_{\xi, y}\right|^{p} \mathrm{~d} t+\mathcal{H}^{0}\left(A_{y}^{\xi} \cap S_{\left(u_{j}\right)_{\xi, y}}\right)\right) \mathrm{d} \mathcal{H}^{n-1}(y) \\
& \quad \geq 2 \int_{A_{\xi}} \mathcal{H}^{0}\left(A_{y}^{\xi} \cap\left(S_{u}\right)_{y}^{\xi} \cap\left(\Sigma_{u}\right)_{y}^{\xi}\right) \mathrm{d} \mathcal{H}^{n-1}(y)=2 \int_{A \cap S_{u} \cap \Sigma_{u}}\left|\left\langle\nu_{u}(x), \xi\right\rangle\right| \mathrm{d} \mathcal{H}^{n-1}(x) .
\end{aligned}
$$

In the last equality we took advantage of the $\mathcal{H}^{n-1}$-countably rectifiability of $S_{u} \cap \Sigma_{u}$ which is inherited by that of $S_{u}$.

Eventually, by passing to the supremum on a countable dense set $\left(\xi_{k}\right)_{k \in \mathbf{N}} \in \mathbf{S}^{n-1}$ in (4.13) and using the monotone convergence theorem we deduce

$$
\liminf _{j} F_{\psi}\left(u_{j}, A\right) \geq 2 \mathcal{H}^{n-1}\left(A \cap S_{u} \cap \Sigma_{u}\right)
$$

which is equivalent to (4.5) thanks to $(2.7)$.

Step 2. Proof of (4.6). We will first prove a localized "rough" version of inequality (4.6) (see (4.15)). To do that we will exploit the auxiliary obstacle function $\psi-u$ outside the jump set of $u$, and use $u_{j}-u$ as admissible test function in the formulation of the related obstacle problem for the Mumford-Shah functional. This estimate will provide straightforward the finiteness of $\sigma\left(A \cap\left(\Sigma_{u} \backslash S_{u}\right)\right)$, which in turn can be used to improve the former inequality and get (4.6).

Notice that this strategy can be exploited only outside the discontinuity set $S_{u}$ of the target function. 
Let $A^{\prime}$ be an open set in $A$. An easy computation shows that for any $\varepsilon>0$ it holds

$$
\begin{aligned}
F_{\psi}\left(u_{j}, A\right) & \geq 2^{1-p} \int_{A^{\prime}}\left|\nabla\left(u_{j}-u\right)\right|^{p} \mathrm{~d} x-\int_{A^{\prime}}|\nabla u|^{p} \mathrm{~d} x+\mathcal{H}^{n-1}\left(A^{\prime} \cap S_{u_{j}}\right) \\
& \geq 2^{1-p} \int_{A^{\prime}}\left|\nabla\left(u_{j}-u\right)\right|^{p} \mathrm{~d} x+\mathcal{H}^{n-1}\left(A^{\prime} \cap S_{u_{j}-u}\right)-M S_{p}\left(u, A^{\prime}\right) \\
& \geq \inf \left\{2^{1-p} \int_{A^{\prime}}|\nabla v|^{p} \mathrm{~d} x+\mathcal{H}^{n-1}\left(A^{\prime} \cap S_{v}\right)+\frac{1}{\varepsilon} \int_{A^{\prime}}|v|^{p} \mathrm{~d} x: v^{+} \geq \psi-\tilde{u} \mathcal{H}^{n-1} \text { a.e. on } A^{\prime} \backslash S_{u}\right\} \\
& -\frac{1}{\varepsilon} \int_{A^{\prime}}\left|u_{j}-u\right|^{p} \mathrm{~d} x-M S_{p}\left(u, A^{\prime}\right),
\end{aligned}
$$

where in the second inequality we used that $S_{u_{j}-u} \subseteq S_{u} \cup S_{u_{j}}$, and in the third that $\left(u_{j}-u\right)^{+}(x)=u_{j}^{+}(x)-\tilde{u}(x) \geq$ $\psi(x)-\tilde{u}(x)$ for $\mathcal{H}^{n-1}$ a.e. $x \in \Omega \backslash S_{u}$.

Passing first to the inferior limit as $j \rightarrow+\infty$, and then taking the supremum on $\varepsilon>0$ in (4.14), by Remark 3.4 and Proposition 3.5 we get

$$
\liminf _{j} F_{\psi}\left(u_{j}, A\right) \geq \sigma\left(\Sigma_{u} \backslash S_{u}, A^{\prime}\right)-M S_{p}\left(u, A^{\prime}\right) .
$$

In particular by choosing $A^{\prime}=A$ in the previous inequality we get $\sigma\left(A \cap\left(\Sigma_{u} \backslash S_{u}\right)\right)<+\infty$ so that $\mathcal{L}^{n}(A \cap$ $\left.\left(\Sigma_{u} \backslash S_{u}\right)\right)=0$ by (2.5). Hence, by the outer/inner regularity of both the Lebesgue measure and the measure associated to $M S_{p}(u, \cdot)$ we can find a sequence of open sets $A_{k} \subseteq A$ such that $A_{k} \supseteq A \cap\left(\Sigma_{u} \backslash S_{u}\right)$ and $\lim _{k} M S_{p}\left(u, A_{k}\right)=0$.

Indeed, for any $k \in \mathbf{N}$ let $C_{k} \subseteq S_{u}$ be a compact set such that $\mathcal{H}^{n-1}\left(S_{u} \backslash C_{k}\right) \leq 1 / k$, and $\delta_{k}>0$ such that $\int_{V}|\nabla u|^{p} \mathrm{~d} x \leq 1 / k$ for any $V \mathcal{L}^{n}$ measurable with $\mathcal{L}^{n}(V) \leq \delta_{k}$. Since $\mathcal{L}^{n}\left(A \cap\left(\Sigma_{u} \backslash S_{u}\right)\right)=0$ one can also find an open set $V_{k}$ such that $A \supseteq V_{k} \supseteq A \cap\left(\Sigma_{u} \backslash S_{u}\right)$ and $\mathcal{L}^{n}\left(V_{k}\right) \leq \delta_{k}$. Then, choose in (4.15) the open sets $A_{k}=V_{k} \backslash C_{k}$ to get

$$
\liminf _{j} F_{\psi}\left(u_{j}, A\right) \geq \sigma\left(\Sigma_{u} \backslash S_{u}, A_{k}\right)-\frac{2}{k}
$$

Then, being $A_{k} \cap\left(\Sigma_{u} \backslash S_{u}\right)=A \cap\left(\Sigma_{u} \backslash S_{u}\right)$, we deduce from Theorem 2.4(b)

$$
\liminf _{j} F_{\psi}\left(u_{j}, A\right) \geq \sigma\left(A \cap\left(\Sigma_{u} \backslash S_{u}\right)\right)-\frac{2}{k} .
$$

Eventually, (4.6) follows by letting $k \rightarrow+\infty$.

We now provide the upper bound inequality.

Proposition 4.3. For every $u \in L^{1}(\Omega)$ there exists $\left(u_{j}\right) \subset L^{1}(\Omega)$ such that $u_{j} \rightarrow u$ in $L^{1}(\Omega)$ and

$$
\limsup _{j} F_{\psi}\left(u_{j}, \Omega\right) \leq \mathcal{F}_{\psi}(u, \Omega) .
$$

Proof. We may assume $\mathcal{F}_{\psi}(u, \Omega)<+\infty$, from which it follows $u \in G S B V(\Omega)$ and $\sigma\left(\Sigma_{u}\right)<+\infty$, where we recall that $\Sigma_{u}=\left\{x \in \Omega: u^{+}(x)<\psi(x)\right\}$.

Let $w \in G S B V(\Omega)$ be such that $F_{\psi}(w, \Omega)<+\infty$. By Remark 2.7 for every $j \in \mathbf{N}$ there exists an open set $\Omega_{j} \subset \Omega$ such that $\Sigma_{u} \cup S_{w} \subseteq \Omega_{j}, \mathcal{L}^{n}\left(\Omega_{j}\right) \leq \frac{1}{j^{2}}$ for $j$ sufficiently big, and

$$
\operatorname{Per}\left(\Omega_{j}, \Omega\right) \leq \sigma\left(\Sigma_{u} \cup S_{w}\right)+\frac{1}{j}
$$


Define $u_{j}=u \chi_{\Omega \backslash \Omega_{j}}+w \chi_{\Omega_{j}}$, it is then easy to check that $u_{j} \rightarrow u$ in $L^{1}(\Omega)$. Furthermore, Theorem 3.84 [2] yields $u_{j} \in G S B V(\Omega)$ and

$$
D u_{j}=D w\left\llcorner\Omega_{j}^{1}+\left(u_{j}^{+}-u_{j}^{-}\right) \nu_{\partial^{*} \Omega_{j}} \mathcal{H}^{n-1}\left\llcorner\partial^{*} \Omega_{j}+D u\left\llcorner\Omega_{j}^{0}\right.\right.\right.
$$

(see Ths. 3.84 and 4.34 [2]), in particular $S_{u_{j}} \subseteq S_{w} \cup \partial^{*} \Omega_{j} \cup\left(S_{u} \cap \Omega_{j}^{0}\right)$. Thus, $u_{j}^{+}=w^{+}$on $\Omega_{j}^{1}, u_{j}^{+}=\tilde{w} \vee u^{+}$ on $\partial^{*} \Omega_{j}$, and $u_{j}^{+}=u^{+}$on $\Omega_{j}^{0}$. Hence, we deduce $u_{j}^{+} \geq \psi \mathcal{H}^{n-1}$ a.e. $\Omega$, by taking into account that $\Sigma_{u} \cup S_{w} \subseteq \Omega_{j}$ and $F_{\psi}(w, \Omega)<+\infty$. Furthermore, Lebesgue differentiation theorem gives $\mathcal{L}^{n}\left(\Omega_{j}^{1} \backslash \Omega_{j}\right)=0$, and then by (4.17) we have

$$
\begin{aligned}
F_{\psi}\left(u_{j}, \Omega\right)= & M S_{p}\left(u_{j}, \Omega\right) \leq \int_{\Omega}|\nabla u|^{p} \mathrm{~d} x+\mathcal{H}^{n-1}\left(S_{u} \backslash \Sigma_{u}\right) \\
& +\int_{\Omega_{j}}|\nabla w|^{p} \mathrm{~d} x+\mathcal{H}^{n-1}\left(S_{w}\right)+\mathcal{H}^{n-1}\left(\partial^{*} \Omega_{j}\right) .
\end{aligned}
$$

Thus, by (4.16) it follows

$$
\limsup _{j} F_{\psi}\left(u_{j}, \Omega\right) \leq \int_{\Omega}|\nabla u|^{p} \mathrm{~d} x+\mathcal{H}^{n-1}\left(S_{u} \backslash \Sigma_{u}\right)+\sigma\left(\Sigma_{u}\right)+\mathcal{H}^{n-1}\left(S_{w}\right)+\sigma\left(S_{w}\right),
$$

from which we infer

$$
\bar{F}_{\psi}(u, \Omega) \leq \mathcal{F}_{\psi}(u, \Omega)+3 \mathcal{H}^{n-1}\left(S_{w}\right) .
$$

In order to take care of the extra energy contribution due to $w$ we notice that $w \vee M \in G S B V(\Omega)$ is such that $F_{\psi}(w \vee M, \Omega)<+\infty$. Thus, we may repeat the construction performed above substituting $w$ with $w \vee M$ in order to get

$$
\bar{F}_{\psi}(u, \Omega) \leq \mathcal{F}_{\psi}(u, \Omega)+3 \mathcal{H}^{n-1}\left(S_{w \vee M}\right),
$$

and thus to conclude it suffices to show that $\mathcal{H}^{n-1}\left(S_{w \vee M}\right)$ is infinitesimal as $M \rightarrow+\infty$.

The $B V$ chain rule formula implies $\mathcal{H}^{n-1}\left(S_{w \vee M}\right)=\mathcal{H}^{n-1}\left(\left\{x \in \Omega: w^{+}(x)>M\right\} \cap S_{w}\right)$ (see Th. 3.101 [2]), and thus by taking into account that $\mathcal{H}^{n-1}\left(\left\{x \in \Omega:\left|w^{ \pm}(x)\right|=+\infty\right\}\right)=0$ (see (4.3)) we infer

$$
\lim _{M \rightarrow+\infty} \mathcal{H}^{n-1}\left(S_{w \vee M}\right)=0
$$

Remark 4.4. The choice to express the unilateral obstacle condition in (4.1) by the representant $u^{+}$can be easily justified in one-dimension. Indeed, in such a case the same conclusion of Theorem 4.1 holds true even using the representant $u^{-}$.

\section{Relaxation for the Dirichlet problem With an obstacle}

In this section we add a Dirichlet boundary value to the obstacle problem. In order to do that we suppose that $\Omega$ is a bounded open set of $\mathbf{R}^{n}$ with Lipschitz boundary.

In such a case, we recall that any $u \in B V(\Omega)$ leaves an inner boundary trace on $\partial \Omega$ denoted by $\operatorname{tr}(u)$, and that the trace operator $\operatorname{tr}: B V(\Omega) \rightarrow L^{1}\left(\partial \Omega, \mathcal{H}^{n-1}\right.$ ) is onto (see Th. $3.87[2]$ ). Moreover, for any $u \in G S B V(\Omega)$ with $M S_{p}(u, \Omega)<+\infty$ one can show that for $\mathcal{H}^{n-1}$ a.e. $x \in \partial \Omega$ the value ap- $\lim _{y \rightarrow x, y \in \Omega} u(y)$ exists finite (see the comments after Th. 4.34 [2] and Prop. 2.4 [11]). We call it the trace of $u$ and denote it still by $\operatorname{tr}(u)$.

With fixed $\varphi \in L^{1}\left(\partial \Omega, \mathcal{H}^{n-1}\right)$ we introduce the functional $D_{\psi, \varphi}: L^{1}(\Omega) \rightarrow[0,+\infty]$ defined as

$$
D_{\psi, \varphi}(u, \Omega)=F_{\psi}(u, \Omega) \text { if } u \in G S B V(\Omega), \operatorname{tr}(u)=\varphi \mathcal{H}^{n-1} \text { a.e. on } \partial \Omega,
$$


$+\infty$ otherwise in $L^{1}(\Omega)$. To ensure the problem to be non trivial we impose a compatibility condition between the trace and obstacle functions: We assume that there exists $w \in L^{p} \cap G S B V(\Omega)$ such that $D_{\psi, \varphi}(w, \Omega)<+\infty$, namely

$$
M S_{p}(w, \Omega)<+\infty, w^{+} \geq \psi \mathcal{H}^{n-1} \text { a.e. in } \Omega, \operatorname{tr}(w)=\varphi \mathcal{H}^{n-1} \text { a.e. on } \partial \Omega .
$$

In addition, given any bounded open set $\widehat{\Omega}$ such that $\widehat{\Omega} \supset \supset$, a local reflection argument shows that we may suppose $w \in G S B V(\widehat{\Omega}), M S_{p}(w, \widehat{\Omega})<+\infty$ and $\mathcal{H}^{n-1}\left(\partial \Omega \cap S_{w}\right)=0$. Hence, if $u \in G S B V(\Omega)$ we denote by $\widehat{u}$ the function obtained extending $u$ by $w$ in $\mathbf{R}^{n} \backslash \Omega$, so that $\widehat{u} \in G S B V(\widehat{\Omega})$, and

$$
M S_{p}(\widehat{u}, \widehat{\Omega})=M S_{p}(u, \Omega)+M S_{p}(w, \widehat{\Omega} \backslash \bar{\Omega})+\mathcal{H}^{n-1}(\{x \in \partial \Omega: \operatorname{tr}(u)(x) \neq \varphi(x)\})
$$

(see Ths. 3.84 and $3.87[2]$ ).

Theorem 5.1. Under the previous assumptions the lower semicontinuous envelope $\mathcal{D}_{\psi, \varphi}: L^{1}(\Omega) \rightarrow[0,+\infty]$ of $D_{\psi, \varphi}$ is given by

$$
\mathcal{D}_{\psi, \varphi}(u, \Omega)=\mathcal{F}_{\psi}(u, \Omega)+\mathcal{H}^{n-1}(\{x \in \partial \Omega: \operatorname{tr}(u)(x) \neq \varphi(x)\})
$$

if $u \in G S B V(\Omega),+\infty$ otherwise in $L^{1}(\Omega)$.

In the proof below we keep the same notation of Propositions 4.2 and 4.3.

Proof. Let $\left(u_{j}\right) \in G S B V(\Omega)$ with $u_{j} \rightarrow u$ in $L^{1}(\Omega)$ and $\liminf D_{\psi, \varphi}\left(u_{j}, \Omega\right)<+\infty$ be given. Then, $u \in$ $\operatorname{GSBV}(\Omega)$ and $\widehat{u}_{j} \rightarrow \widehat{u}$ in $L^{1}(\widehat{\Omega})$. Furthermore, if $\widehat{\psi}$ is the extension of $\psi$ by $-\infty$ in $\widehat{\Omega} \backslash \Omega$, we may apply Theorem 4.1 to $F_{\widehat{\psi}}$ on $\widehat{\Omega}$ and get by (5.2) above

$$
\begin{aligned}
\liminf _{j} D_{\psi, \varphi}\left(u_{j}, \Omega\right) & =\liminf _{j} F_{\widehat{\psi}}\left(\widehat{u}_{j}, \widehat{\Omega}\right)-M S_{p}(w, \widehat{\Omega} \backslash \bar{\Omega}) \\
& \geq \mathcal{F}_{\widehat{\psi}}(\widehat{u}, \widehat{\Omega})-M S_{p}(w, \widehat{\Omega} \backslash \bar{\Omega})=\mathcal{D}_{\psi, \varphi}(u, \Omega) .
\end{aligned}
$$

To prove the upper bound inequality for $u \in G S B V(\Omega)$ such that $\mathcal{D}_{\psi, \varphi}(u, \Omega)<+\infty$, we consider a sequence $\left(A_{j}\right)$ of open sets such that $E \subset A_{j}, \lim _{j} \mathcal{L}^{n}\left(A_{j}\right)=0$ and $\lim _{j} \operatorname{Per}\left(A_{j}\right)=\sigma(E)$, being $E=\{x \in \partial \Omega: \operatorname{tr}(u)(x) \neq$ $\varphi(x)\}$. Thanks to Theorem 2.4 (b) the sequence $\left(A_{j}\right)$ can be chosen in an arbitrarily small neighbourhood of $\partial \Omega$.

Then, we define a sequence $\left(v_{j}\right)$ performing the same construction of Proposition 4.3 replacing $\Omega_{j}$ there with $\Omega_{j} \cup A_{j}$, and $w$ there with the function satisfying condition (5.1). Arguing as in Proposition 4.3 it is easy to check that $v_{j} \rightarrow u$ in $L^{1}(\Omega)$, and $v_{j}^{+} \geq \psi \mathcal{H}^{n-1}$ a.e. in $\Omega$. Furthermore, $\operatorname{tr}\left(v_{j}\right)=w$ on $\partial \Omega$, so that for any open set $A \subset \subset \Omega$ we get

$$
\begin{aligned}
& D_{\psi, \varphi}\left(v_{j}, \Omega \backslash \bar{A}\right) \leq \int_{\Omega \backslash \bar{A}}|\nabla u|^{p} \mathrm{~d} x+\mathcal{H}^{n-1}\left(\left(S_{u} \backslash \Sigma_{u}\right) \cap(\Omega \backslash \bar{A})\right) \\
& \quad+\int_{\Omega_{j} \cup A_{j}}|\nabla w|^{p} \mathrm{~d} x+\mathcal{H}^{n-1}\left(S_{w} \cap(\Omega \backslash \bar{A})\right)+\operatorname{Per}\left(\Omega_{j}, \Omega \backslash \bar{A}\right)+\operatorname{Per}\left(A_{j}, \Omega\right) .
\end{aligned}
$$

By (4.16) and the definition of $\sigma$ it is easy to see that if $\mathcal{H}^{n-1}\left(\partial A \cap\left(\Sigma_{u} \cup S_{w}\right)\right)=0$ it holds

$$
\lim _{j} \operatorname{Per}\left(\Omega_{j}, \Omega \backslash \bar{A}\right)=\sigma\left(\left(\Sigma_{u} \cup S_{w}\right) \cap(\Omega \backslash \bar{A})\right) .
$$

In addition, in Lemma 5.3 below we will show that $\lim _{j} \operatorname{Per}\left(A_{j}, \Omega\right)=\mathcal{H}^{n-1}(E)$, taken this for granted we have

$$
\limsup _{j} D_{\psi, \varphi}\left(v_{j}, \Omega \backslash \bar{A}\right) \leq \mathcal{D}_{\psi, \varphi}(u, \Omega \backslash \bar{A})+3 \mathcal{H}^{n-1}\left(S_{w} \cap(\Omega \backslash \bar{A})\right) .
$$


To take care of the extra energy contribution due to $w$ we refine the construction above as we did in Proposition 4.3. In this case we have to take also into account that the boundary datum cannot be changed.

To this aim choose open sets $U_{1} \subset \subset U_{2} \subset \subset \Omega$ with $\mathcal{H}^{n-1}\left(\partial U_{k} \cap\left(\Sigma_{u} \cup S_{w}\right)\right)=0, k=1,2$, and let $\eta \in C_{0}^{\infty}\left(U_{2}\right)$ be a smooth cut-off function such that $\left.\eta\right|_{U_{1}} \equiv 1$. Denote by $u_{j}$ the function constructed as $v_{j}$ above with $w \vee M$ in place of $w$. We remark that we may also assume $\mathcal{H}^{n-1}\left(\partial U_{k} \cap S_{v_{j}}\right)=\mathcal{H}^{n-1}\left(\partial U_{k} \cap S_{u_{j}}\right)=0$ for $j \in \mathbf{N}$ and $k=1,2$.

Set $w_{j}=(1-\eta) v_{j}+\eta u_{j}$, then $w_{j} \rightarrow u$ in $L^{1}(\Omega)$. By construction $u_{j} \geq v_{j} \mathcal{L}^{n}$ a.e. in $\Omega$, then $w_{j} \geq v_{j} \mathcal{L}^{n}$ a.e. in $\Omega$ which in turn implies $w_{j}^{+} \geq v_{j}^{+} \geq \psi \mathcal{H}^{n-1}$ a.e. in $\Omega$. Furthermore, $\operatorname{tr}\left(w_{j}\right)=\varphi$ on $\partial \Omega$ and a straightforward computation yields

$$
\begin{aligned}
D_{\psi, \varphi}\left(w_{j}, \Omega\right) & \leq D_{\psi, \varphi}\left(v_{j}, \Omega \backslash \overline{U_{1}}\right)+F_{\psi}\left(u_{j}, U_{2}\right)+\|\nabla \eta\|_{L^{\infty}(\Omega)}^{p} \int_{U_{2} \backslash \overline{U_{1}}}\left|v_{j}-u_{j}\right|^{p} \mathrm{~d} x \\
& \leq D_{\psi, \varphi}\left(v_{j}, \Omega \backslash \overline{U_{1}}\right)+F_{\psi}\left(u_{j}, U_{2}\right)+\|\nabla \eta\|_{L^{\infty}(\Omega)}^{p} \int_{\Omega_{j} \cup A_{j}}|w-M|^{p} \mathrm{~d} x .
\end{aligned}
$$

By assumption $w \in L^{p}(\Omega)$, thus by taking into account (4.18) and (5.3) we can pass to the limit in the inequality above and get

$$
\bar{D}_{\psi, \varphi}(u, \Omega) \leq \mathcal{D}_{\psi, \varphi}(u, \Omega)+\mathcal{F}_{\psi}\left(u, U_{2} \backslash \overline{U_{1}}\right)+3 \mathcal{H}^{n-1}\left(S_{w} \cap\left(\Omega \backslash \overline{U_{1}}\right)\right)+3 \mathcal{H}^{n-1}\left(S_{w \vee M} \cap U_{2}\right),
$$

the conclusion then follows by letting first $M \rightarrow+\infty$ (see (4.3)) and then shrinking $\Omega \backslash \overline{U_{1}}$ to $\emptyset$.

Remark 5.2. It is easy to check that the functional $\mathcal{D}_{\psi, \varphi}$ is not coercive on $L^{1}(\Omega)$. In order to ensure compactness for its sublevel sets one has to add a lower order term to fulfil the assumptions of the $G S B V$ compactness Theorem 2.1.

To conclude the section we are left with proving the following result.

Lemma 5.3. For any $\mathcal{H}^{n-1}$ measurable set $E \subseteq \partial \Omega$, and any sequence $\left(A_{j}\right)$ of open sets such that $E \subset A_{j}$, $\lim _{j} \mathcal{L}^{n}\left(A_{j}\right)=0$ and $\lim _{j} \operatorname{Per}\left(A_{j}\right)=\sigma(E)$, we have

$$
\lim _{j} \operatorname{Per}\left(A_{j}, \Omega\right)=\mathcal{H}^{n-1}(E) .
$$

Proof. First we recall that if $A$ is an open set with Lipschitz continuous boundary the $L^{1}$ lower semicontinuous envelope of $L^{1}(A) \ni u \rightarrow\|D u\|(A)$ if $\operatorname{tr}(u)=\chi_{E} \mathcal{H}^{n-1}$ a.e. on $\partial A$ is given by

$$
\|D u\|(A)+\int_{\partial A}\left|\operatorname{tr}(u)-\chi_{E}\right| \mathrm{d} \mathcal{H}^{n-1}
$$

if $u \in B V(A),+\infty$ otherwise in $L^{1}(A)$ (see [3]). Then, since $E \subset A_{j}$ and $\chi_{A_{j}} \rightarrow 0$ in $L^{1}\left(\mathbf{R}^{n}\right)$, choosing $A=\Omega$ yields

$$
\liminf _{j} \operatorname{Per}\left(A_{j}, \Omega\right)=\liminf _{j}\left\|D \chi_{A_{j}}\right\|(\Omega) \geq \int_{\partial \Omega}\left|\chi_{E}\right| \mathrm{d} \mathcal{H}^{n-1}=\mathcal{H}^{n-1}(E) .
$$

We can repeat the same argument with $\mathbf{R}^{n} \backslash \bar{\Omega}$ in place of $\Omega$ and get

$$
\underset{j}{\liminf } \operatorname{Per}\left(A_{j}, \mathbf{R}^{n} \backslash \bar{\Omega}\right) \geq \mathcal{H}^{n-1}(E) .
$$


Hence, we deduce

$$
\begin{aligned}
\sigma(E) & =2 \mathcal{H}^{n-1}(E)=\lim _{j} \operatorname{Per}\left(A_{j}\right) \\
& \geq \limsup _{j} \operatorname{Per}\left(A_{j}, \Omega\right)+\liminf _{j} \operatorname{Per}\left(A_{j}, \mathbf{R}^{n} \backslash \bar{\Omega}\right) \geq \limsup _{j} \operatorname{Per}\left(A_{j}, \Omega\right)+\mathcal{H}^{n-1}(E),
\end{aligned}
$$

and the thesis follows.

\section{FurTher RESUlts}

In this section we extend the relaxation result obtained in Section 4 for the Mumford-Shah energy with an obstacle to more general free-discontinuity energies. We limit ourselves to introduce all the ingredients needed for the generalization of Theorem 4.1 in the new setting, being the proof a straightforward extension.

We consider the functional $F: L^{1}(\Omega) \rightarrow[0,+\infty]$

$$
F(u, \Omega)=\int_{\Omega} f(\nabla u) \mathrm{d} x+\int_{S_{u}} \varphi\left(\nu_{u}\right) \mathrm{d} \mathcal{H}^{n-1}
$$

for $u \in G S B V(\Omega),+\infty$ otherwise; where $f, \varphi: \mathbf{R}^{n} \rightarrow \mathbf{R}$ are continuous functions satisfying:

(a) $f$ is convex, and there exist constants $c_{1}, c_{2}>0$ such that for every $\xi \in \mathbf{R}^{n}$

$$
c_{1}|\xi|^{p} \leq f(\xi) \leq c_{2}|\xi|^{p}
$$

(b) $\varphi$ is a norm on $\mathbf{R}^{n}$.

According to Theorem $2.1 F$ turns out to be $L^{1}$ lower semicontinuous.

With a fixed Borel function $\psi: \mathbf{R}^{n} \rightarrow \mathbf{R} \cup\{ \pm \infty\}$ we consider the obstacle functional $F_{\psi}: L^{1}(\Omega) \rightarrow[0,+\infty]$ related to $F$ and $\psi$ by

$$
F_{\psi}(u, \Omega)=F(u, \Omega) \quad u \in G S B V(\Omega), u^{+} \geq \psi \mathcal{H}^{n-1} \text { a.e. on } \Omega
$$

$+\infty$ otherwise in $L^{1}(\Omega)$.

To determine the relaxation $\overline{F_{\psi}}$ of $F_{\psi}$ we introduce the set functions $\sigma_{\varphi}^{\varepsilon}(\cdot, A)$ given by

$$
\inf \left\{\int_{\partial^{*} D \cap A} \varphi\left(\nu_{\partial^{*} D}\right) \mathrm{d} \mathcal{H}^{n-1}+\frac{1}{\varepsilon} \mathcal{L}^{n}(D \cap A): D \mathcal{L}^{n} \text {-measurable, } \mathcal{H}^{n-1}\left(E \backslash D_{+}\right)=0\right\},
$$

and $\sigma_{\varphi}(E, A)=\sup _{\varepsilon>0} \sigma_{\varphi}^{\varepsilon}(E, A)$. Notice that in case $\varphi$ is the Euclidean norm we recover De Giorgi's measure $\sigma$. The new set function $\sigma_{\varphi}$ is obtained by replacing the usual perimeter with the anisotropic perimeter defined through $\varphi$ in the definition of $\sigma$. Analogous statements to those in Proposition 2.4 hold true for $\sigma_{\varphi}$ (see Props. 4.3 and $4.6[6]$ ); in particular $\sigma_{\varphi}$ is a regular Borel measure.

Therefore, by arguing as in Section 3 and Section 4 we deduce the following result.

Theorem 6.1. Let $F_{\psi}$ be as in (6.1), then its lower semicontinuous envelope in the $L^{1}$ topology is given by

$$
\begin{aligned}
\mathcal{F}_{\psi}(u, \Omega)= & \int_{\Omega} f(\nabla u) \mathrm{d} x+\int_{S_{u}} \varphi\left(\nu_{u}\right) \mathrm{d} \mathcal{H}^{n-1} \\
& +\frac{1}{2} \sigma_{\varphi}\left(\left\{x \in S_{u}: u^{+}(x)<\psi(x)\right\}\right)+\sigma_{\varphi}\left(\left\{x \in \Omega \backslash S_{u}: u^{+}(x)<\psi(x)\right\}\right)
\end{aligned}
$$

if $u \in G S B V(\Omega),+\infty$ otherwise in $L^{1}(\Omega)$.

Eventually, we remark that if a Dirichlet boundary datum is imposed a result similar to Theorem 5.1 holds true. 


\section{REFERENCES}

[1] L. Ambrosio and A. Braides, Energies in SBV and variational models in fracture mechanics, in Homogenization and Applications to Material Sciences, D. Cioranescu, A. Damlamian and P. Donato Eds., GAKUTO, Gakkōtosho, Tokio, Japan (1997) 1-22.

[2] L. Ambrosio, N. Fusco and D. Pallara, Functions of Bounded Variation and Free Discontinuity Problems. Oxford University Press, Oxford (2000).

[3] G. Anzellotti, The Euler equation for functionals with linear growth. Trans. Amer. Math. Soc. 290 (1985) 483-501.

[4] A. Braides, Approximation of Free-Discontinuity Problems, Lecture Notes in Mathematics. Springer-Verlag, Berlin (1998).

[5] A. Braides, $\Gamma$-convergence for beginners. Oxford University Press, Oxford (2002).

[6] M. Carriero, G. Dal Maso, A. Leaci and E. Pascali, Relaxation of the non-parametric Plateau problem with an obstacle. J. Math. Pures Appl. 67 (1988) 359-396.

[7] M. Carriero, G. Dal Maso, A. Leaci and E. Pascali, Limits of obstacle problems for the area functional, in Partial Differential Equations and the Calculus of Variations, Vol. I, PNDEA 1, Birkhäuser Boston, Boston (1989) 285-309.

[8] F. Colombini, Una definizione alternativa per una misura usata nello studio di ipersuperfici minimali. Boll. Un. Mat. Ital. 8 (1973) 159-173.

[9] G. Dal Maso, An Introduction to Г-convergence. Birkhäuser, Boston (1993).

[10] G. Dal Maso, Variational problems in Fracture Mechanics. Preprint S.I.S.S.A. (2006).

[11] G. Dal Maso, G. Francfort and R. Toader, Quasistatic crack growth in nonlinear elasticity. Arch. Ration. Mech. Anal. 176 (2005) 165-225.

[12] E. De Giorgi, Problemi di superfici minime con ostacoli: forma non cartesiana. Boll. Un. Mat. Ital. 8 (1973) 80-88.

[13] E. De Giorgi and L. Ambrosio, Un nuovo funzionale del calcolo delle variazioni. Atti Accad. Naz. Lincei Rend. Cl. Sci. Fis. Mat. Natur. 82 (1988) 199-210.

[14] E. De Giorgi, F. Colombini and L.C. Piccinini, Frontiere orientate di misura minima e questioni collegate. Quaderno della Scuola Normale Superiore di Pisa, Editrice Tecnico Scientifica, Pisa (1972).

[15] M. Focardi and M.S. Gelli, Asymptotic analysis of Mumford-Shah type energies in periodically perforated domains. Interfaces and Free Boundaries 9 (2007) 107-132.

[16] J.E. Hutchinson, A measure of De Giorgi and others does not equal twice the Hausdorff measure. Notices Amer. Math. Soc. 24 (1977) A-240.

[17] J.E. Hutchinson, On the relationship between Hausdorff measure and a measure of De Giorgi, Colombini, Piccinini. Boll. Un. Mat. Ital. 18-B (1981) 619-628.

[18] D. Mumford and J. Shah, Optimal approximation by piecewise smooth functions and associated variational problems. Comm. Pure Appl. Math. 17 (1989) 577-685.

[19] L.C. Piccinini, De Giorgi's measure and thin obstacles, in Geometric measure theory and minimal surfaces, C.I.M.E. III Ciclo, Varenna (1972) 221-230; Edizioni Cremonese, Rome (1973). 